\title{
Disordered Elastic Metasurfaces
}

Liyun $\mathrm{Cao}^{1,2}$, Zhichun Yang ${ }^{1 *}$, Yanlong Xu${ }^{1}$, Shi-Wang Fan², Yifan Zhu², Zhaolin Chen ${ }^{1}$, Brice

Vincent $^{2}$ and Badreddine Assouar ${ }^{2 *}$

${ }^{1}$ School of Aeronautics, Northwestern Polytechnical University, Xi'an, 710072, China

${ }_{2}^{2}$ Institut Jean Lamour, CNRS, Université de Lorraine, Nancy, 54506, France

*Corresponding author e-mails: yangzc@nwpu.edu.cn \& badreddine.assouar@univ-lorraine.fr

\begin{abstract}
The discovery of the disorder effect in traditional metamaterials has opened the possibilities in the search for the disordered metasurfaces. Photonic, dielectric and elastic metamaterials exhibiting the added value of the disorder effect on wave propagation physics, have been reported. Despite this extensive attention and progress in disordered metamaterials, the elastic metasurfaces however, involving disorder have not yet been reported. Here, we introduce the concept of disordered elastic metasurface composed of identical pillared resonators with a random arrangement in the subwavelength range. Based on theoretical formalism and direct acoustic measurement, we observe anomalous deflection and focusing effects of flexural waves in a plate, and elucidate in details the related physics. This research extends the disorder effect to metasurfaces and may lead to innovative acoustoelastic devices.
\end{abstract}

Keywords: Elastic metasurfaces, metamaterials, disorder, wave manipulation 


\section{INTRODUCTION}

Since the emergence of metamaterials, the study of the disorder has become an important research direction [1-3]. A deep understanding of disorder effect can help to improve metamaterials properties [4,5] and enrich their physics [6-9]. For instance, the disorder can widen bandgap in elastic metamaterials [5] and induce topological state transition in photonic metamaterials [8] or phase transitions in dielectric metamaterials [9]. In contrast, independent of the spatial organization of the material, the invariant band gaps of metamaterials can also be observed by taking advantage of strong localization effects [10]. As a consequence, a detailed analysis of wave properties in disordered metamaterial is of interest, not only for physics, but also for pragmatic applications. Recently, as one kind of ultrathin metamaterials [11-13], optic metasurfaces $[14,15]$ and acoustic metasurfaces [16-19] have been proposed to realize extraordinary wave manipulation. For elastic waves, due to complex wave coupling, the elastic metasurface design $[20,21]$ is of paramount significance to the current research efforts in a large variety of applications ranging from ultrasonic detection to vibration control. Very recently, disorder had been introduced to the optic metasurface [22], which exhibits additional unique features for wavefront shaping such as a tailorable angular scattering profile. However, the acoustic/elastic metasurfaces involving disorder have not yet been reported.

In this research, we introduce the concept of disordered elastic metasurface (DEM) composed of identical pillared resonators with a random arrangement in the subwavelength range. We analytically and numerically reveal the decoupled region of 
the pillared resonators. The phase shifts and transmission amplitudes of the subunits are only linearly related to the number of resonators and independent of the spatial arrangement of resonators. The disorder releases the degree of freedom of pillar position in the subunit, which can greatly enhance the flexibility for the reconfigurable subunit. Both numerical simulations and experiments are carried out to demonstrate the extraordinary functionalities of DEM, including anomalous deflection and focusing. The physics of the disorder in the proposed metasurface can be regarded as a universal method for transmissive metasurfaces and metastructure designs. The DEM may also have a direct pragmatic variety of applications in the field of controllable wave manipulation and related acoustoelastic devices and wave engineering.

\section{MODEL AND DESIGN}

Fig. 1(a) shows the schematic of DEM with predesigned geometries comprising an array of subunits, which is capable of manipulating flexural wave in a plate. The three-dimensional (3D) subunit is constructed based on pillared resonators in Fig. 1(b). The corresponding two-dimensional (2D) model of the subunit is depicted in Fig. 1(c). All the attached resonators have the same geometrical parameter: thickness $d_{2}$ and height $h$ are $3 \mathrm{~mm}$ and $30 \mathrm{~mm}$, respectively. The resonators are attached on the plate with a disordered arrangement, i.e., the spacing $s_{j}$ between the $j$ th resonator and $(j+1)$ th resonator is arbitrary. Considering both flexural and longitudinal waves propagate in the hosting plate and resonators, the coefficient vectors of the wave fields at the point P of the incident region and the point Q of the transmitted region (marked in Fig. 1(c)) 
are denoted as $\mathbf{k}_{\text {in }}=\left[1, r_{b}, 0, r_{b}^{*}, 0, r_{l}\right]^{T}$ and $\mathbf{k}_{\text {out }}=\left[t_{b}, 0, t_{b}^{*}, 0, t_{l}, 0\right]^{T}$ (see the details in Appendix A), respectively. $r_{b}, t_{b}, r_{b}^{*}, t_{b}^{*}, r_{l}$, and $t_{l}$ are the amplitude ratios of the reflected propagating flexural wave, transmitted propagating flexural wave, reflected evanescent flexural wave, transmitted evanescent flexural wave, reflected propagating longitudinal wave, and transmitted propagating longitudinal wave to the incident propagating flexural wave, respectively.

(a)

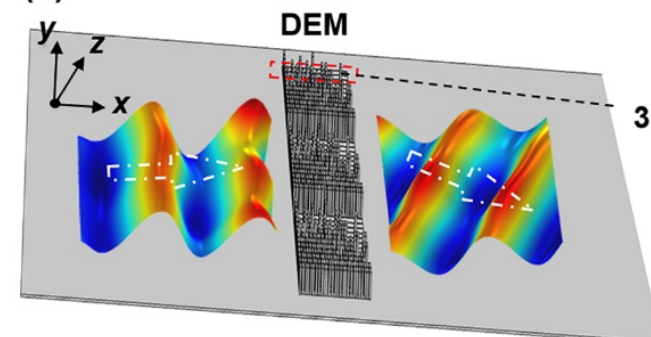

(b)

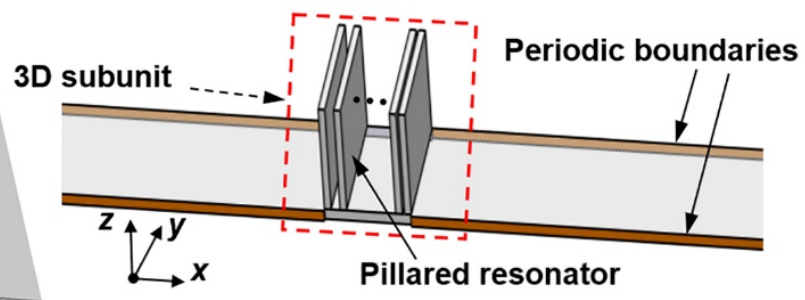

(c)

$5 \lambda$

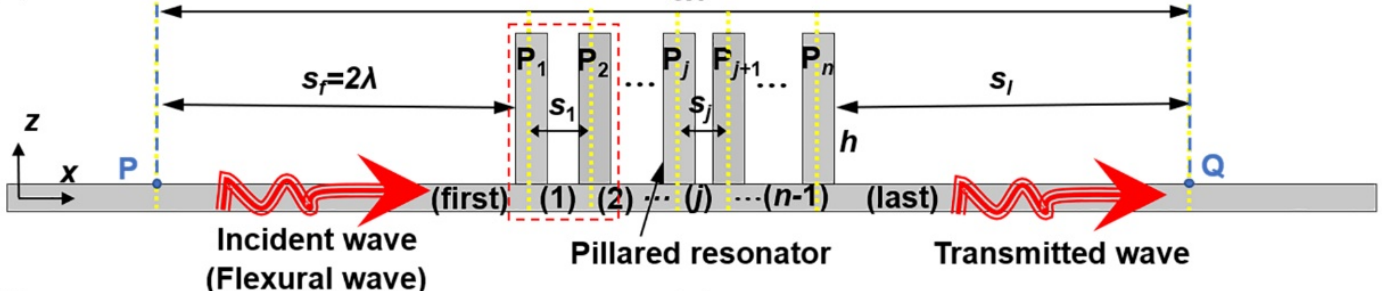

(d)

(e)
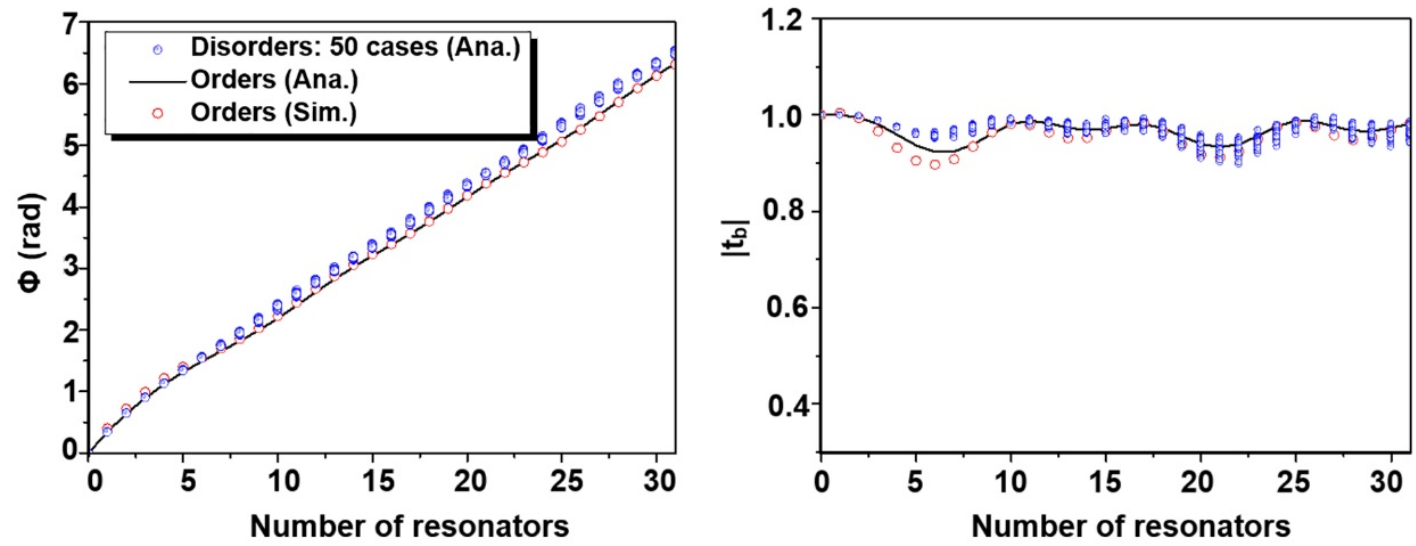

FIG. 1. Disordered elastic metasurface (DEM). (a) Schematic diagram of DEM. (b) Three-

dimensional (3D) view of a subunit. (c) Cross-section view of a subunit. (d), (e) The phase shift and transmission amplitude solved analytically and numerically for disordered and ordered resonators as function of the number of resonators in the subwavelength subunit. 
The transfer equation for disordered multiresonator shown in Fig. 1(c) (the detailed derivation can be found in Appendix A) is

$$
\mathbf{k}_{\text {out }}=N_{2}^{(\text {last })} N_{1} N_{2}^{(n-1)} N_{1} \cdots N_{2}^{(2)} N_{1} N_{2}^{(1)} N_{1} N_{2}^{(\text {first })} \mathbf{k}_{\text {in }}
$$

where $n$ is the number of the pillared resonator, $N_{1}$ is the transfer matrix for incident wave propagating from the left to the right of the pillared resonator. $N_{2}^{(1)}, N_{2}^{(2)} \quad \cdots$ and $N_{2}^{(n-1)}$ are the transfer matrixes for the flexural and longitudinal waves propagating in the plate regions with pillar spacing $s_{1}, s_{2} \cdots$ and $s_{n-1}$, respectively. $N_{2}^{\text {(first) }}$ and $N_{2}^{\text {(last) }}$ are the transfer matrixes for waves propagating in Region first and Region last, respectively. For the subunit with different number of resonators, we randomly adjust the pillar spacing $s_{j}$ between the $j$ th resonator and $(j+1)$ th resonator, which meets the relation of $n \cdot d_{2}<\sum_{j=1}^{n-1} s_{j}<\lambda$ to make the total multiresonator thickness not exceed one wavelength $\lambda$, i.e., sub-wavelength. In this way, for the different number of attached resonators, 50 disordered subunits are randomly built without loss of generality. To calculate the phase shift and transmission amplitude analytically, we assume that the pillared resonators and plate are made of aluminum alloy. The excitation frequency is selected as $1 \mathrm{kHz}$ and the corresponding flexural wave wavelength for the plate with a thickness of $3 \mathrm{~mm}$ is 57.1 times the pillar thickness $\left(d_{2}=3 \mathrm{~mm}\right)$. The transmission amplitude $\left|t_{b}\right|$ at the point $\mathrm{Q}$ is calculated analytically from Eq. (A6), and the corresponding phase shift is extracted from the complex transmission coefficient $t_{b}$ (see the details in Appendix A).

In Figs. 1(d) and 1(e), the analytical results of the phase shift and transmission amplitude of the flexural waves across the disordered subunits are plotted as blue circles. 
Several interesting phenomena can be observed as follows. First, randomly disrupting the arrangement of resonators almost does not affect the phase shift and transmission amplitude. In other words, the phase shift and transmission amplitude are only related to the number of resonators in the subunits, but independent of the arrangement. It indicates that the designed metasurface has stronger robustness than conventional designs. Second, the phase shifts by varying the number of resonators can span over a full phase range of $2 \pi$ with high transmission amplitude (bigger than 0.9). This ensures the high-efficiency of the metasurface for fine field manipulations [23,24]. Third, the phase shift increases linearly with the number of resonators. This allows us to simply predict the phase shift of these subunits with the different number of resonators.

Furthermore, the ordered arrangements of resonators are also investigated for comparison, with all the pillars spacing keep uniformly $5 \mathrm{~mm}$ without loss of generality. The transmission coefficient $t_{b}$ can be calculated analytically from the modified transfer equation $\mathbf{k}_{\text {out }}=N_{2}^{\text {(last) }} N_{1}\left(N_{2}^{*} N_{1}\right)^{n-1} N_{2}^{(\text {first }} \mathbf{k}_{\text {in }}$ (see the details in Appendix A), where $N_{2}^{*}$ is the transfer matrix for the wave propagating in the plate region of the identical pillars spacing. The phase shift and the transmission amplitude by varying the number of resonators are solved analytically and numerically (plotted as black solid lines and red circles ) in Figs.1(d) and 1(e). The simulated results are in good agreement with the analytical ones, which confirms the accuracy of the analytical method. Meanwhile, the results for the ordered resonators are very close to the disordered ones, showing the introduction of the disorder will not affect the phase shift and transmission amplitude. 


\section{THE PHYSICS OF DECOUPLED DISORDERED SUBUNITS}

In order to explain these phenomena, we have investigated the model for two adjacent resonators in the subunit, as marked with the red dotted box in Fig. 1(c). Specifically, we have analyzed the influence of the excitation frequency $f$, the typical structural parameter $h$ (pillar height), and the pillars spacing $s_{1}$ on the phase $\phi_{1}$ and transmission amplitude $\left|t_{1}\right|$ of the point Q in Fig. 1(c). The remaining parameters are the same as those discussed above. The phase $\phi_{1}$ and transmission amplitude $\left|t_{1}\right|$ can be expressed as $\phi_{1}=G_{\phi 1}\left(f, h, s_{1}\right)$ and $\left|t_{1}\right|=G_{|t 1|}\left(f, h, s_{1}\right)$, respectively. From the above analysis of the disorder unit, it can be seen that under certain conditions, the phase and transmission amplitude are independent of the pillars spacing. In order to give a quantitative evaluation of the dependence of phase and transmission amplitude on the pillars spacing, we define the coupling strengths as:

$$
\begin{aligned}
\Lambda_{\phi_{1}, s_{1}}\left(f, h, s_{1}\right) & =\frac{\partial G_{\phi_{1}}\left(f, h, s_{1}\right)}{\partial s_{1}} \\
\Lambda_{\left|t_{1}\right| s_{1}}\left(f, h, s_{1}\right) & =\frac{\partial G_{\left|t_{1}\right|}\left(f, h, s_{1}\right)}{\partial s_{1}}
\end{aligned}
$$

We further obtain the coupling coefficients $\bar{\Lambda}_{\phi_{1}, s_{1}}(f, h)$ and $\bar{\Lambda}_{\left|t_{1}\right| s_{1}}(f, h)$ by integrating the coupling strengths for all different pillars spacing $(0.003 \lambda, \lambda)$ and conducting normalization with respect to their maxima. The effects of changing $f$ and $h$ on the coupling coefficients $\bar{\Lambda}_{\phi_{1}, s_{1}}$ and $\bar{\Lambda}_{\left|t_{1}\right|, s_{1}}$ are shown analytically in Figs. 2(a) and 2(b), respectively. It clearly shows the existence of completely decoupled regions characterized by $\bar{\Lambda}_{\phi_{1}, s_{1}}=0$ and $\bar{\Lambda}_{\left|t_{1}\right|, s_{1}}=0$, respectively, which means that the phase shift and the transmission amplitude are independent of the pillars spacing. The marked 
decoupled boundary lines of the phase and transmission amplitude are almost at the same location.

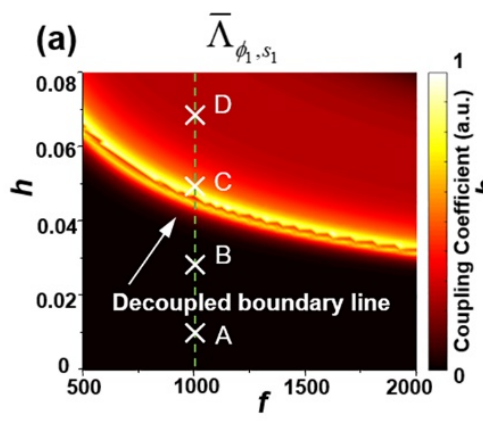

(d)

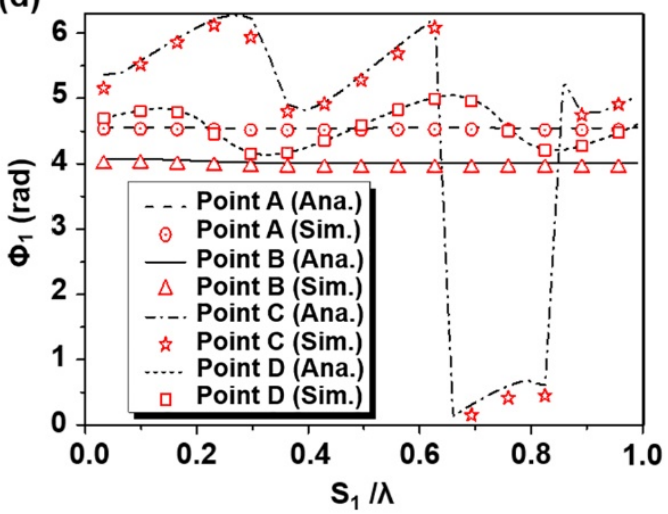

(b) $\quad \bar{\Lambda}_{\left|t_{1}\right|, s_{1}}$

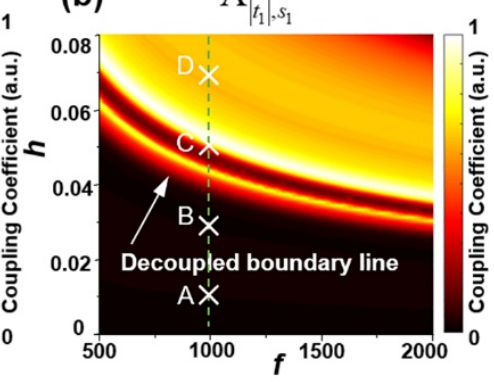

(c)

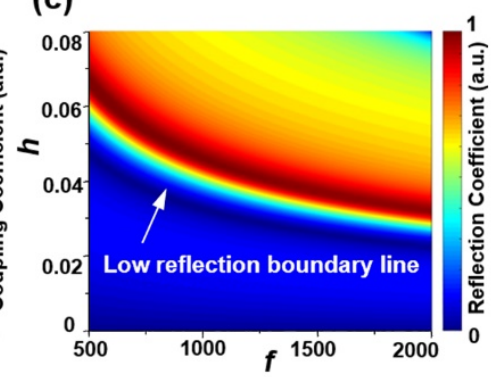

(e)

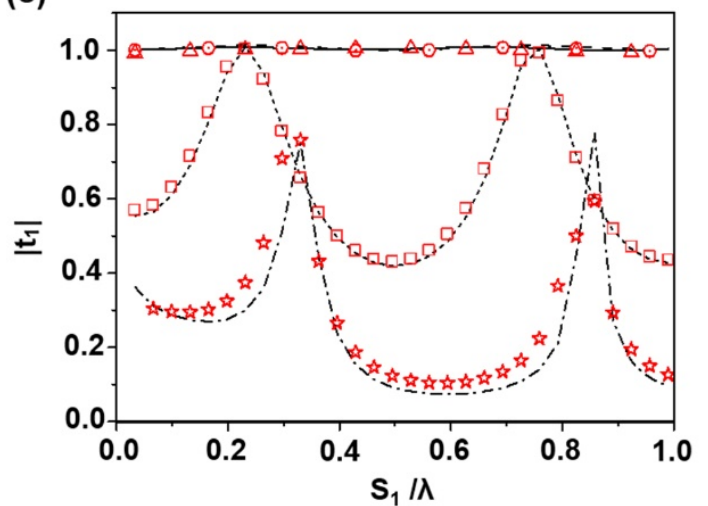

FIG. 2. The physics of decoupled disordered resonators. (a), (b) Effect of the changes of $f$ and $h$ on the analytical coupling coefficients $\bar{\Lambda}_{\phi_{1}, s_{1}}$ and $\bar{\Lambda}_{\left|t_{1}\right|, s_{1}}$, respectively. (c) Analytical reflection coefficient for a single resonator by varying the excitation frequency $f$ and resonator height $h$. For the points $\mathrm{A}, \mathrm{B}, \mathrm{C}$, and $\mathrm{D}$ in (a) and (b), the phase and transmission amplitude in the far field (the point Q) by varying the pillars spacing are analytically and numerically shown in (d) and (e), respectively.

To further reveal the underlying physics, the reflection coefficient of a single resonator by varying the excitation frequency $f$ and the resonator height $h$ is analytically obtained (see the details in Appendix A), as shown in Fig. 2(c). We label the low reflection boundary line and observe that the decoupled boundary lines for the phase and transmission amplitude are consistent with the low reflection boundary line. This 
indicates that the decoupling of the phase and transmission amplitude with respect to the pillars spacing depends on the low reflection intensity of the incident wave pass through the resonator. Therefore, the low reflection coupling between resonators induced from their low reflection intensity will lead to decoupling, releasing the degree of freedom of pillar position in the subunit. It should be pointed out that Fabry-Perot resonance, where the phases equal to an integer multiple of $\pi$, also has a low reflection intensity. However, it does not lead to the above decoupling. The proposed physics can broaden the understanding of metamaterials and be applied to other transmissive metasurfaces for optics, acoustics, and elastics.

For intuitively displaying the above analysis conclusions, predictive decoupled points A and B and coupling points $C$ and D are chosen from Figs. 2(a) and 2(b). For these four points, the phase and transmission amplitude in the far field as function of the pillars spacing are solved analytically and numerically. The results are shown in Figs. 2(d) and 2(e). One can observe that the phase and transmission amplitude at the points $\mathrm{A}$ and $\mathrm{B}$ are independent of the pillars spacing, while at the points $\mathrm{C}$ and $\mathrm{D}$, they are strongly dependent on. These results confirm the validity of our theoretical prediction. The point $\mathrm{B}$ in the decoupled region corresponds to the above disordered subunit analyzed in Figs. 1(d) and 1(e).

\section{DEM MANIPULATING THE FLEXURAL WAVE}

The above designed 2D disordered subunits can be extended directly to the corresponding three-dimensional (3D) ones with slots (see in Appendix B), while the 
phase shift and transmission amplitude almost remain unchanged. The elastic metasurfaces are designed by the 3D disordered subunits. The different number of the pillared resonators are attached on the hosting plate with slots (see in Appendix C) to achieve different function, including anomalous deflection and focusing. Importantly, these resonators can be conveniently disassembled and reattached, since the disordered and ordered resonators have the same effect on wave manipulation. It is convenient to continuously change the phase shift for every subunit by changing their number of resonators. It should be pointed out that the reconfigurable subunit can be designed (the detailed design can be found in Appendix D) based on the disordered subunit. The disorder can greatly enhance the flexibility for the reconfigurable subunit, because it releases the degree of freedom of pillar position in the subunit.

\section{A. Simulations and experiments of the anomalous deflections}

The DEM with the functions of anomalous deflection is designed. The corresponding individual supercells are shown in the right of Figs. 3(a) and 3(b). They are composed of eight types of chosen disordered 3D subunits, composed of $0,2,6,11$, $15,19,23,27$ identical pillared resonators respectively, in the random arrangement. These subunits can make transmitted waves with phase shifts cover a full range of $2 \pi$ and high transmittance according to Figs. 1(d) and 1(e). The discrete phase shift of the transmitted wave increases linearly along the $y$-axis, and the spatial phase shift gradients for Figs. 3(a) and 3(b) are $d \phi / d y=0.5 k$ and $d \phi / d y=k$, respectively, where $k=2 \pi / \lambda$. According to the generalized Snell's law [14], for the vertically and obliquely 
(the incident angle of $30^{\circ}$ ) incident waves, the theoretical refraction angles are $30^{\circ}$ and $-30^{\circ}$, respectively. Full-wave simulations (detailed geometric parameters of the units and more associated simulation details are provided in the Appendixes $\mathrm{C}$ and $\mathrm{E}$ ) clearly demonstrate the anomalous deflections. The ordered elastic metasurface is also constructed for comparison, shown in the Appendixes F. The metasurfaces with the subunits possessing the required phase shifts are fabricated and shown in Figs. 3(c) and 3(d). The full field experimental measurements including the incident and transmitted fields at different time points are shown in Figs. 3(e) and 3(f), which are in good agreement with both the analytical predictions and simulated results. The detailed propagation phenomena are presented in Movie S1 and Movie S2 of the Supplemental Material [25].

(a) Disordered pillars

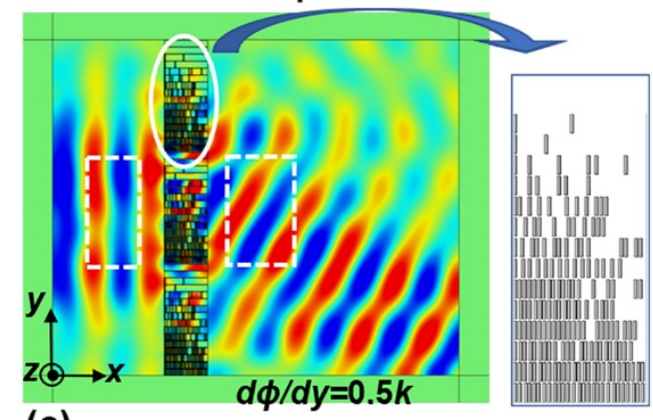

(c)

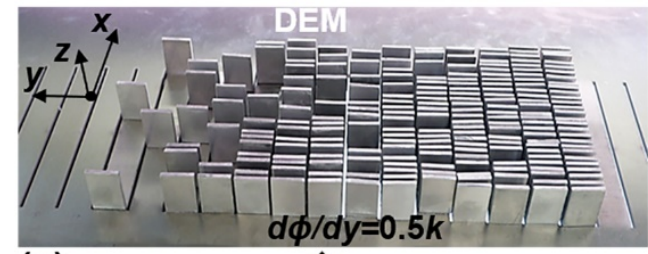

(e)

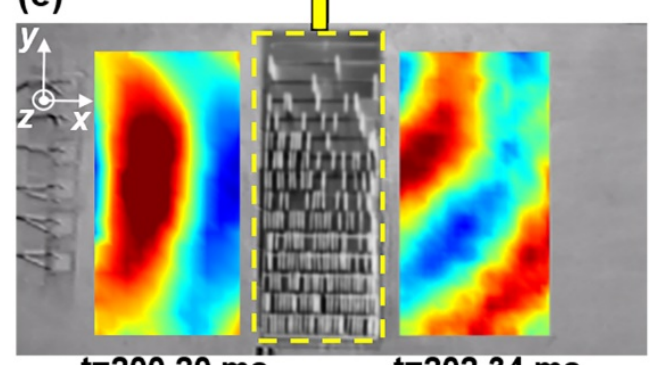

$\mathrm{t}=\mathbf{2 0 0} .20 \mathrm{~ms}$ $\mathrm{t}=202.34 \mathrm{~ms}$ (b) Disordered pillars

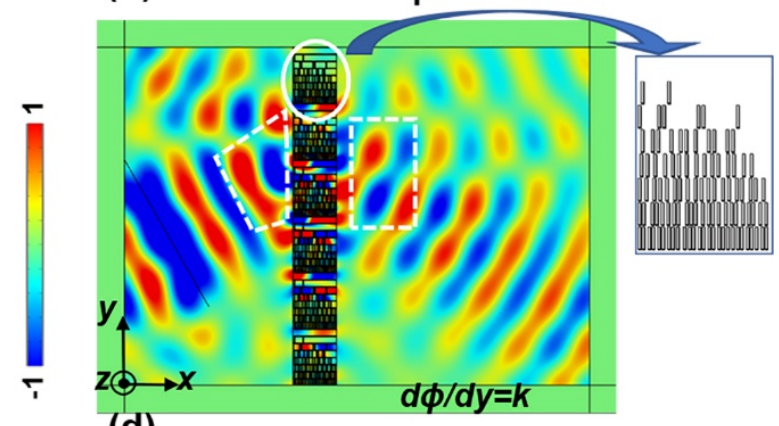

(d)

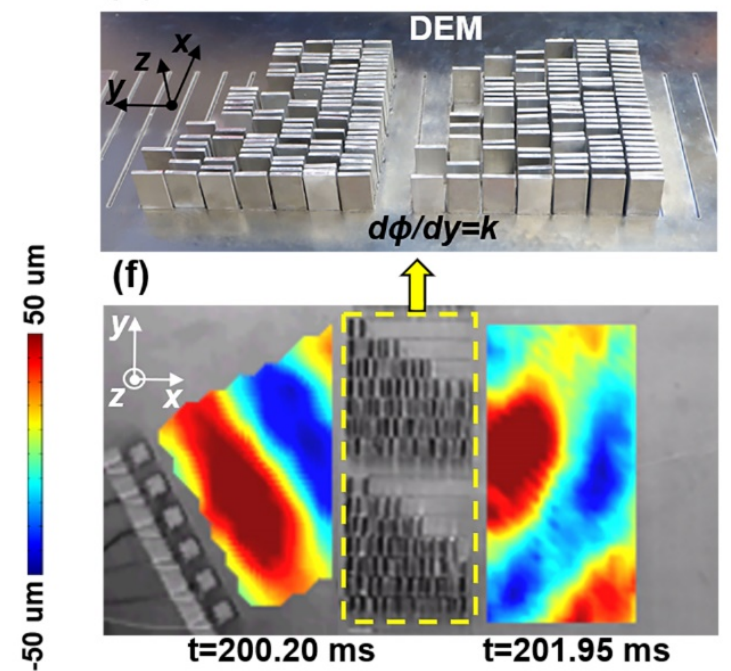


FIG. 3. Deflecting flexural wave with DEM. (a), (b) The wave fields for vertically and obliquely incident Gaussian beams pass through two disordered metasurfaces with phase shift gradients of $d \phi / d y=0.5 k$ and $d \phi / d y=k$, respectively. (c), (d) The fabricated metasurfaces. (e), (f) The full field experimental measurements including the incident and transmitted fields at different time points. The incident and the transmitted fields of (e) are measured at $200.20 \mathrm{~ms}$ and $202.34 \mathrm{~ms}$, respectively.

\section{B. Simulation and experiment of the focusing}

By employing the disordered subunits, our metasurface design can also be exploited to achieve flexural waves focusing, as shown in Fig. 4(e). The DEM can be readily constructed by selecting units that match the requested hyperbolic phase profile $\phi(y)=\frac{2 \pi}{\lambda}\left(\sqrt{F^{2}+y^{2}}-F\right)$, where $F=1.5 \lambda$ is the desired focal distance (detailed geometric parameters of the DEM are provided in the Appendixes C). The transmitted intensity and displacement fields with focus at $1.5 \lambda$ are simulated and shown in Figs. 4(a) and 4(c), respectively. One clearly observes a focal spot after the incident waves pass through the DEM. The full field experimental measurement is shown in Fig. 4(d). The detailed propagation phenomenon is presented in Movie S3 of the Supplemental Material [25]. For a more quantitative comparison, the normalized intensity fields of the transmitted wave at the focal spot along the $y$-direction from the simulations and experiments are shown in Fig. 4(b). The sharp peak values are observed, which are about more than 3 times the intensity of the incident wave. The experimental measurement is in good agreement with the simulated result and demonstrates a fine 
focusing capability.

(a)

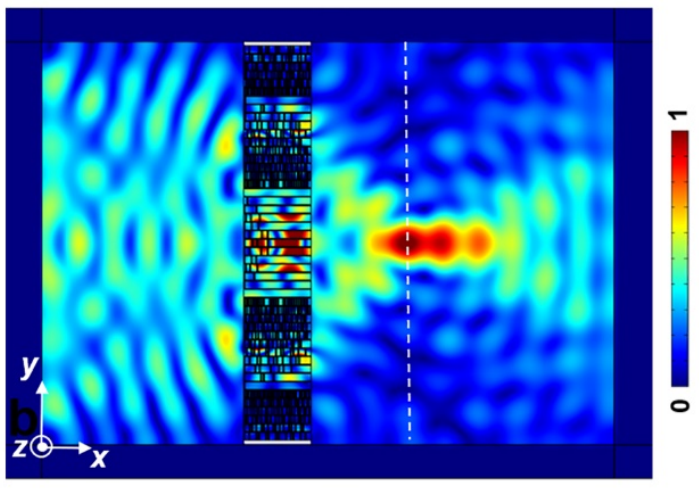

(b)

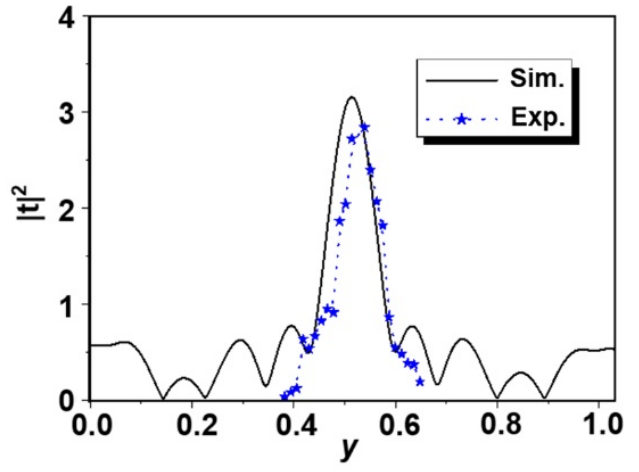

(e)

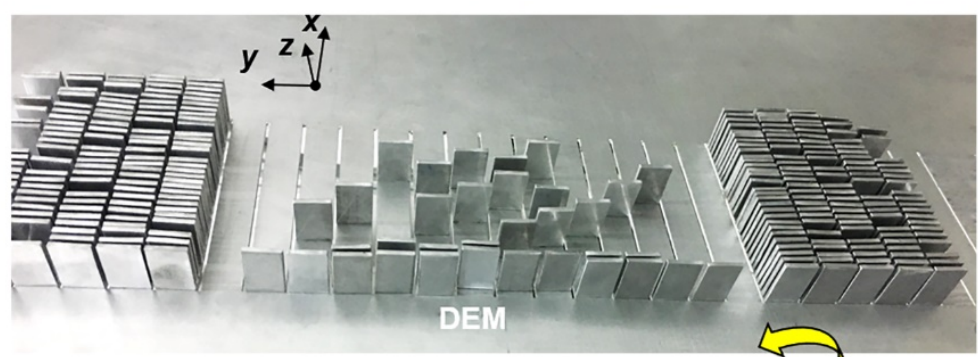

(c)

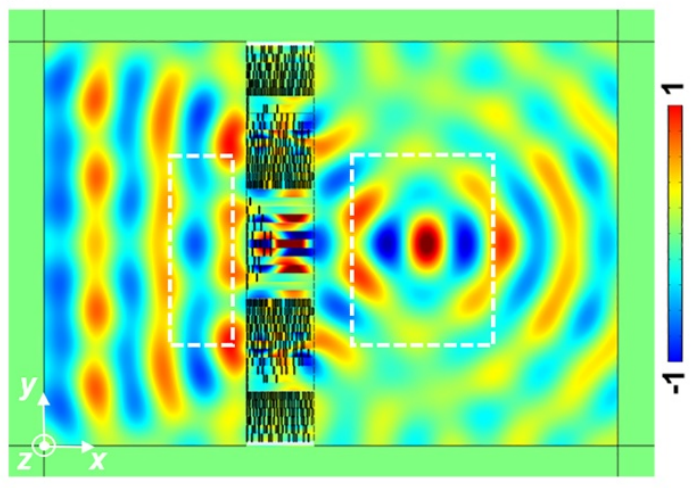

(d)

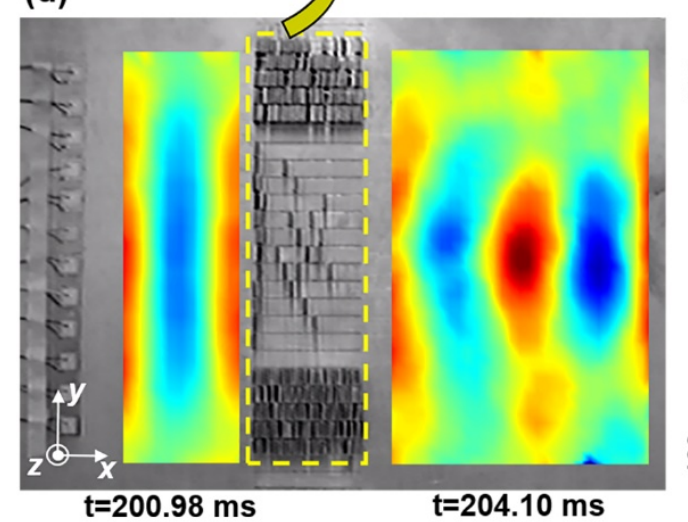

FIG. 4. Focusing flexural wave with DEM. (a), (c) The simulated results of the transmitted intensity and displacement fields with focus at $1.5 \lambda$ for the DEM, respectively. (b) The normalized transmitted intensity fields at the focal spot along the $y$-direction from the simulation and experiment. (e) The fabricated structure of the DEM. (d) The full field experimental measurement.

\section{CONCLUSION}

In summary, we have reported a feature of elastic metasurfaces composed of 
identical pillared resonators. By reducing the reflection coupling between resonators below the low reflection boundary line in parameter space, the dependence of resonators in the subwavelength subunits on the spatial arrangement can be adjusted from existence to absence. In the decoupled region of the disordered resonators, the transmitted waves pass through the subunits with approximately full transmission, while the phase shift can be continuously adjusted by the number of resonators in the disordered spatial arrangements. In addition, we performed full wave experimental measurement of DEM structures to demonstrate the multifunction, including anomalous deflection and focusing. Our findings are of significance importance in understanding the physics of elastic waves interaction with disordered artificial media. The present work may open venues for a variety of applications in the field of controllable wave manipulation and related acoustoelastic devices.

\section{ACKNOWLEDGMENT}

This work was supported by the project CARNOT ICEEL "METACOM" and la Région Grand Est, by National Natural Science Foundation of China (Grant No. 11972296 and No. 11602194), by the 111 Project (Grant No. BP0719007), and by

Natural Science Basic Research Plan in Shaanxi Province of China (Grant No. 2018JQ1076). The first author is grateful to the support of Innovation Foundation for Doctor Dissertation of Northwestern Polytechnical University (Grant No. CX201936) and China Scholarship Council (CSC Grant No. 201806290176). 


\section{APPENDIX A: ANALYTICAL SOLUTION FOR DISORDERED AND}

\section{ORDERED SUBUNITS}

\section{Analytical modeling}

The host plate in the subunit can be divided into several regions, which are marked as Regions first, $1,2 \ldots j \ldots n-1$ and last in Fig. 1(c). The governing equations for the flexural wave and the longitudinal wave in the plate can be expressed as the following forms, respectively:

$$
\begin{aligned}
& E_{1} I_{1} \frac{\partial^{4} w(x, t)}{\partial x^{4}}+\rho_{1} d_{1} \frac{\partial^{2} w(x, t)}{\partial t^{2}}=0 \\
& \frac{E_{1}}{\left(1-v_{1}^{2}\right)} \frac{\partial^{2} u(x, t)}{\partial x^{2}}-\rho_{1} \frac{\partial^{2} u(x, t)}{\partial t^{2}}=0
\end{aligned}
$$

where $E_{1}$ is Young's modulus of the hosting plate, $\rho_{1}$ is the density of the plate and $I_{1}$ is the moment of area of the plate. $I_{1}=\frac{d_{1}^{3}}{12\left(1-v_{1}^{2}\right)}$, in which $d_{1}$ and $v_{1}$. are the thickness and Poisson's ratio of the plate, respectively.

The governing equation of flexural waves in Region $j$ is a fourth-order partial derivative equation. The wave number has four solutions, i.e., two real wave numbers and two complex wave numbers. The real and complex wave numbers represent propagating flexural and evanescent flexural waves, respectively. Therefore, the general solution of displacement for the governing equation is:

$$
w^{(j)}(x, t)=\left(A^{(j)} e^{-i k_{b 1} x}+B^{(j)} e^{i k_{b 1} x}+C^{(j)} e^{-k_{b 1} x}+D^{(j)} e^{k_{b 1} x}\right) e^{i \omega t}
$$

where the superscript $(j)$ indicates Region $j . A^{(j)}, B^{(j)}, C^{(j)}$ and $D^{(j)}$ are complex coefficients. $A^{(j)} e^{-i k_{b 1} x}$ and $B^{(j)} e^{i k_{b 1} x}$ correspond to the positive-going and negativegoing propagating flexural waves, respectively. $C^{(j)} e^{-k_{b 1} x}$ and $D^{(j)} e^{k_{b 1} x}$ correspond to 
the positive-going and negative-going evanescent flexural waves, respectively. The flexural wavenumber is $k_{b 1}=\left(\frac{\rho_{1} d_{1} \omega^{2}}{E_{1} I_{1}}\right)^{1 / 4}$, in which $\omega=2 \pi f$ is the circular frequency. The general solution of the displacement for the second-order partial derivative governing equation of longitudinal waves in the Region $j$ is:

$$
u^{(j)}(x, t)=\left(P^{(j)} e^{-i k_{l 1} x}+Q^{(j)} e^{i k_{l 1} x}\right) e^{i \omega t}
$$

where the longitudinal wavenumber is $k_{l 1}=\left[\frac{\rho_{1} \omega^{2}\left(1-v_{1}^{2}\right)}{E_{1}}\right]^{1 / 2}, P^{(j)} e^{-i k_{l 1} x}$ and $Q^{(j)} e^{i k_{l_{1} x}}$ correspond to the positive-going and negative-going propagating longitudinal waves, respectively.

The governing equations for the flexural wave and the longitudinal wave in the pillared resonator is similar to that in the host plate. The general solutions of the displacements for the flexural wave and longitudinal wave equations in the pillared resonator $j$ are

$$
\left\{\begin{array}{l}
w^{\left(\mathrm{P}_{j}\right)}(z, t)=\left(A^{\left(\mathrm{P}_{j}\right)} e^{-i k_{b 2} z}+B^{\left(\mathrm{P}_{j}\right)} e^{i k_{b 2} z}+C^{\left(\mathrm{P}_{j}\right)} e^{-k_{b 2} z}+D^{\left(\mathrm{P}_{j}\right)} e^{k_{b 2} z}\right) e^{i \omega t} \\
u^{\left(\mathrm{P}_{j}\right)}(z, t)=\left(P^{\left(\mathrm{P}_{j}\right)} e^{-i k_{l 2} z}+Q^{\left(\mathrm{P}_{j}\right)} e^{i k_{l 2} z}\right) e^{i \omega t}
\end{array}\right.
$$

, respectively. The superscript $\left(\mathrm{P}_{j}\right)$ indicates the pillared resonator $j$. The flexural and longitudinal wavenumbers are $k_{b 2}=\left(\frac{\rho_{2} d_{2} \omega^{2}}{E_{2} I_{2}}\right)^{1 / 4}$ and $k_{l 2}=\left[\frac{\rho_{2} \omega^{2}\left(1-v_{2}^{2}\right)}{E_{2}}\right]^{1 / 2}$, respectively. $E_{2}, \rho_{2}$, and $I_{2}$ are the Young's modulus, density, and area moment of inertia of the pillared resonator, respectively. $I_{2}=\frac{d_{2}^{3}}{12\left(1-v_{2}^{2}\right)}$, in which $d_{2}$ and $v_{2}$ are the thickness of the resonator and Poisson's ratio of the material, respectively.

The relationships between slope $\varphi$ and the displacement $w$, between shear force 
$V$ and the displacement $w$, between bending moment $M$ and the displacement $w$, between axial force $F$ and the displacement $u$ are:

$$
\left\{\begin{array}{l}
\varphi=w^{\prime} \\
V=-E I w^{\prime \prime \prime} \\
M=E I w^{\prime \prime} \\
F=\frac{E d}{\left(1-v^{2}\right)} u^{\prime}
\end{array}\right.
$$

, respectively. Among the right interface of Region $j-1$, the left interface of Region $j$, and the lower interface of the resonator $j$, the following boundary conditions must be satisfied:

1. Displacement continuity in $x$ and $z$ directions:

$$
\left\{\begin{array}{l}
u^{(j-1)}=u^{(j)}=-w^{\left(\mathrm{P}_{j}\right)} \\
w^{(j-1)}=w^{(j)}=u^{\left(\mathrm{P}_{j}\right)}
\end{array}\right.
$$

2. Slope continuity:

$$
\frac{\partial w^{(j-1)}}{\partial x}=\frac{\partial w^{(j)}}{\partial x}=\frac{\partial w^{\left(\mathrm{P}_{j}\right)}}{\partial z}
$$

3. Force balance in $x$ and $z$ directions:

$$
\left\{\begin{array}{l}
\frac{E_{1} d_{1}}{\left(1-v_{1}^{2}\right)} \frac{\partial u^{(j-1)}}{\partial x}-\frac{E_{1} d_{1}}{\left(1-v_{1}^{2}\right)} \frac{\partial u^{(j)}}{\partial x}-E_{2} I_{2} \frac{\partial^{3} w^{\left(\mathrm{P}_{j}\right)}}{\partial z^{3}}=0 \\
-E_{1} I_{1} \frac{\partial^{3} w^{(j-1)}}{\partial x^{3}}+E_{1} I_{1} \frac{\partial^{3} w^{(j)}}{\partial x^{3}}-\frac{E_{2} d_{2}}{\left(1-v_{2}^{2}\right)} \frac{\partial u^{\left(\mathrm{P}_{j}\right)}}{\partial z}=0
\end{array}\right.
$$

4. Moment balance:

$$
E_{1} I_{1} \frac{\partial^{2} w^{(j-1)}}{\partial x^{2}}-E_{1} I_{1} \frac{\partial^{2} w^{(j)}}{\partial x^{2}}-E_{2} I_{2} \frac{\partial^{2} w^{\left(\mathrm{P}_{j}\right)}}{\partial z^{2}}=0
$$

5. Forces and moment are 0 at the free boundary of the resonator $j$ : 


$$
-\left.E_{2} I_{2} \frac{\partial^{3} w^{\left(\mathrm{P}_{j}\right)}}{\partial z^{3}}\right|_{z=h}=\left.\frac{E_{2} d_{2}}{\left(1-v_{2}^{2}\right)} \frac{\partial u^{\left(\mathrm{P}_{j}\right)}}{\partial z}\right|_{z=h}=\left.E_{2} I_{2} \frac{\partial^{2} w^{\left(\mathrm{P}_{j}\right)}}{\partial z^{2}}\right|_{z=h}=0
$$

\section{The transfer equation for the disordered multiresonator}

The complex coefficients $A^{(j)}, B^{(j)}, C^{(j)}, D^{(j)}, P^{(j)}, Q^{(j)}$ for Region $(j)$ in the plate can be organized as a vector

$$
\mathbf{k}^{(j)}=\left\{A^{(j)}, B^{(j)}, C^{(j)}, D^{(j)}, P^{(j)}, Q^{(j)}\right\}^{T}
$$

Among the right interface of Region $j-1$, the left interface of Region $j$ and the lower interface of resonator $j$, substituting Eqs. (A2)-(A4) into the boundary conditions, i.e., Eqs. Erreur ! Source du renvoi introuvable.-(A10), we obtain

$$
\mathbf{k}_{L}^{(j)}=N_{1} \mathbf{k}_{R}^{(j-1)}
$$

where $\mathbf{k}_{R}^{(j-1)}$ and $\mathbf{k}_{L}^{(j)}$ indicate the coefficient vectors in the right interface of Region $j-1$ and the left interface of Region $j$, respectively. $N_{1}$ is the transfer matrix for waves propagating from the left to the right of the pillared resonator $j$, and it has included the contribution of the pillared resonators.

The relationship between the vector $\mathbf{k}_{R}^{(j)}$ at the right interface of Region $j$ and the vector $\mathbf{k}_{L}^{(j)}$ at the left interface of Region $j$ can be expressed as follows:

$$
\mathbf{k}_{R}^{(j)}=\left.N_{2}\right|_{s=s_{j}} \mathbf{k}_{L}^{(j)}=N_{2}^{(j)} \mathbf{k}_{L}^{(j)}
$$

where $N_{2}=\left(\begin{array}{cccccc}e^{-i k_{b 1} \cdot s} & 0 & 0 & 0 & 0 & 0 \\ 0 & e^{i k_{b 1} \cdot s} & 0 & 0 & 0 & 0 \\ 0 & 0 & e^{-k_{b 1} \cdot s} & 0 & 0 & 0 \\ 0 & 0 & 0 & e^{k_{b 1} \cdot s} & 0 & 0 \\ 0 & 0 & 0 & 0 & e^{-i k_{11} \cdot s} & 0 \\ 0 & 0 & 0 & 0 & 0 & e^{i k_{11} \cdot s}\end{array}\right)$ is the transfer matrix for waves propagating between the two ends of Region $j$.

The exciting point is assumed as the point $\mathrm{P}$ on the plate surface as shown in Fig. 
1(c). For Region first with length $s_{\mathrm{f}}=2 \lambda$, the vector $\mathbf{k}_{R}^{\text {(first) }}$ at the right interface and the vector $\mathbf{k}_{\text {in }}$ in the left interface of Region first can be expressed as:

$$
\mathbf{k}_{R}^{\text {(first) }}=\left.N_{2}\right|_{s=2 \lambda} \cdot \mathbf{k}_{\text {in }}=N_{2}^{(\text {first })} \cdot \mathbf{k}_{\text {in }}
$$

The examined point for the transmission phase and amplitude is fixed at the point $\mathrm{Q}$, as shown in Fig. 1(c). For Region last with the length $s_{l}$, the vector $\mathbf{k}_{\text {out }}$ at the right end of Region last and the vector $\mathbf{k}_{L}^{\text {(last) }}$ in the left end of Region last can be expressed as:

$$
\mathbf{k}_{\text {out }}=\left.N_{2}\right|_{s=s_{l}^{*}} \cdot \mathbf{k}_{L}^{\text {(last) }}=N_{2}^{(\text {last })} \cdot \mathbf{k}_{L}^{\text {(last) }}
$$

where, $s_{l}^{*}=s_{l}-0.08 n d_{2} \approx 3 \lambda-\sum_{j=1}^{n-1}\left(s_{j}+0.08 d_{2}\right)$ is the modified length of Region last. According to Eqs. (A12)-(A15), for waves propagating from the left interface of Region first to the right interface of Region last, the transfer relationship can be expressed as:

$$
\mathbf{k}_{\text {out }}=N_{2}^{(\text {last })} N_{1} N_{2}^{(n-1)} N_{1} \cdots N_{2}^{(2)} N_{1} N_{2}^{(1)} N_{1} N_{2}^{(\text {first })} \mathbf{k}_{\text {in }}
$$

From this relation, we can solve the transmission amplitude for the flexural wave transmitting through these $n$ resonators. To do this, we make an incident positive-going flexural wave of $1 \cdot e^{i \omega t}$ at the left interface of Region first, so the wave fields at the left interface of Region first and the right interface of Region last can be expressed as follows:

$$
\left\{\begin{array}{l}
w_{L}^{\text {(first) }}(x, t)=\left(1+r_{b}+r_{b}^{*}\right) e^{i \omega t} \\
u_{L}^{\text {(first) }}(x, t)=r_{l} e^{i \omega t} \\
w_{R}^{\text {(last) }}(x, t)=\left(t_{b}+t_{b}^{*}\right) e^{i \omega t} \\
u_{R}^{\text {(last) }}(x, t)=t_{l} e^{i \omega t}
\end{array}\right.
$$

where $r_{b}, t_{b}, r_{b}^{*}, t_{b}^{*}, r_{l}$, and $t_{l}$ are the amplitude ratios of the reflected propagating flexural wave, transmitted propagating flexural wave, reflected evanescent flexural 
wave, transmitted evanescent flexural wave, reflected longitudinal wave, and transmitted longitudinal wave to the incident positive-going propagating flexural wave, respectively. Therefore, the vectors $\mathbf{k}_{\text {in }}$ and $\mathbf{k}_{\text {out }}$ can be written as:

$$
\left\{\begin{array}{l}
\mathbf{k}_{\text {in }}=\left[1, r_{b}, 0, r_{b}^{*}, 0, r_{l}\right]^{\mathrm{T}} \\
\mathbf{k}_{\text {out }}=\left[t_{b}, 0, t_{b}^{*}, 0, t_{l}, 0\right]^{\mathrm{T}}
\end{array}\right.
$$

The transfer equation for the single resonator can be expressed as:

$$
\mathbf{k}_{\text {out }}=N_{2}^{\text {(last) }} N_{1} N_{2}^{\text {(first) }} \mathbf{k}_{\text {in }}
$$

\section{The transfer equation for the ordered multiresonator}

For the ordered subunits, all the pillar spacings keep uniformly $s_{j}=5 \mathrm{~mm}$. The corresponding transfer matrix for wave propagating from the left interface to the right interface of the uniform Region $j$ is written as $N_{2}^{*}=\left.N_{2}\right|_{s=0.005}$. For waves propagating from the left interface of Region first to the right interface of Region last in the ordered subunit, the transfer equation can be expressed as:

$$
\mathbf{k}_{\text {out }}=N_{2}^{(\text {last })} N_{1}\left(N_{2}^{*} N_{1}\right)^{n-1} N_{2}^{(\text {first }} \mathbf{k}_{\text {in }}
$$

\section{Analytical solution for transmission phase shift and amplitude}

According to Eqs. (A16), (A19) and (A20), $r_{b}, r_{b}^{*}, r_{l}, t_{b}, t_{b}^{*}$, and $t_{l}$ can be solved with the aid of MATLAB code. $\left|t_{b}\right|$ is the amplitude ratio between the transmitted propagating flexural wave and the incident propagating flexural wave. The phase can be obtained from the complex amplitude ratio $t_{b}$. The phase shift is obtained by subtracting the phase at the point $\mathrm{Q}$ for the plate with the resonators from that for the plate without resonators. It can be calculated by the following formula: 


$$
\phi= \begin{cases}\pi+\arctan \left(\frac{\operatorname{Imag}\left(t_{b}\right)}{\operatorname{Real}\left(t_{b}\right)}\right) & \text { if } \operatorname{real}\left(t_{b}\right)<0 \\ \arctan \left(\frac{\operatorname{Imag}\left(t_{b}\right)}{\operatorname{Real}\left(t_{b}\right)}\right) & \text { if } \operatorname{real}\left(t_{b}\right)>0\end{cases}
$$

This formula will make the value of the phase shift from 0 to $2 \pi$.

\section{APPENDIX B: DESIGN FROM THE 2D SUBUNIT TO THE 3D ONE}

The above designed subunit is embodied in its $2 \mathrm{D}$ model. To manipulate flexural waves, the subunit should be designed as the corresponding 3D one in Fig. 5(a), which can be formed by simply stretching the 2D model in Fig. 1(c). The stretched width is denoted as $w_{1}$. Because the metasurface is formed by periodically integrating different 3D subunits, the 3D subunit should be analyzed by applying the periodic boundary conditions on the two long boundaries in the 3D strip-like model in Fig. 5(a). Therefore, the formed 3D subunit is equivalent to the corresponding 2D one in Fig. 1(c). However, slots should be cut from the 3D subunit to separate adjacent different 3D subunits in the integrated metasurface, as shown in Fig. 5(b). The aim is to avoid the Fano interference [26] between the adjacent different 3D subunits to independently design the subunits.

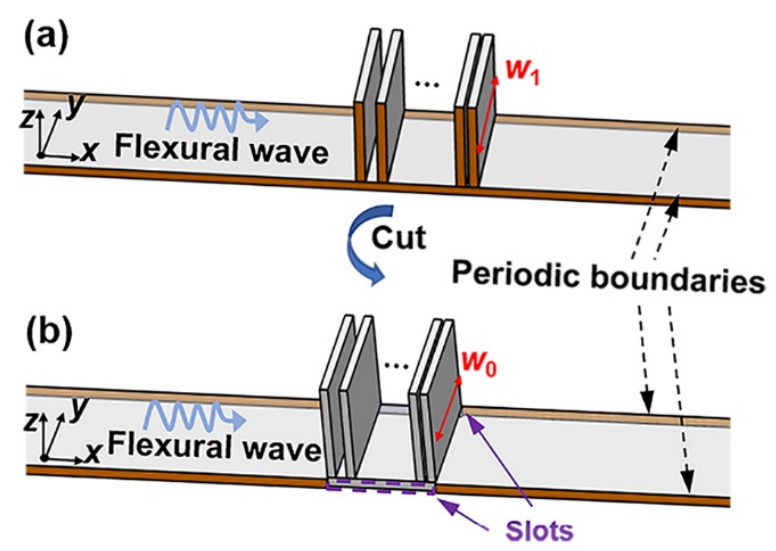

FIG. 5. (a) Model diagram of the 3D subunit without slots. (b) Model diagram of the 3D subunit with slots. 
The filling ratio of the slots in one subunit is denoted as $\eta$, which is defined as dividing the slot width by the stretched width $w_{1}$ in a subunit. In our designs, the widths of the processed pillared resonator $w_{0}$ and the slot are $20.4 \mathrm{~mm}$ and $1 \mathrm{~mm}$, respectively, i.e., $w_{1}=21.4$ and $\eta=0.047$. The slots are very small relative to the stretched width of the subunit. The little slot will lead to a very small flexural wave scattering, which will not affect the transmission amplitude and phase shift [27]. Therefore, the 3D subunit structure with the slot still can be equivalent to the corresponding $2 \mathrm{D}$ one.

\section{APPENDIX C: GEOMETRY OF FABRICATED DEM AND TEST SET-UP}

To accurately fabricate the metasurface, the Computer Numerical Control (CNC) milling machine with a manufacturing precision of $0.01 \mathrm{~mm}$ was adopted. The DEMs in the experimental measurements are composed of the identical processed resonator. For the processed plate, slots should be cut to separate adjacent different 3D subunits in a metasurface, as shown in Figs. 6-8. The adjacent slots make up many strip-like models. Different numbers of the processed resonators are attached to the strip-like model to make up the different subunits, which consist of the metasurface. The numbers in yellow fonts represent the different number of attached resonators in the strip-like model, as shown in Figs. 6-8. These resonators can be conveniently disassembled and reattached, because the glue layer is very thin, in addition, there is no need for the ordered arrangement of these resonators. This process enables to design the disordered metasurface to achieve tunable multifunction by using one type of pillared resonator and the same hosting plate. For example, the fabricated metasurface in Fig. 6 can 
achieve anomalous deflection (the experimental result shows in Figs. 3(e)), the ones in Fig. 7 and Fig. 8 can achieve negative deflection (the experimental result shows in Figs.

3(f) ) and focusing (the experimental result shows in Fig. 4(d)), respectively.

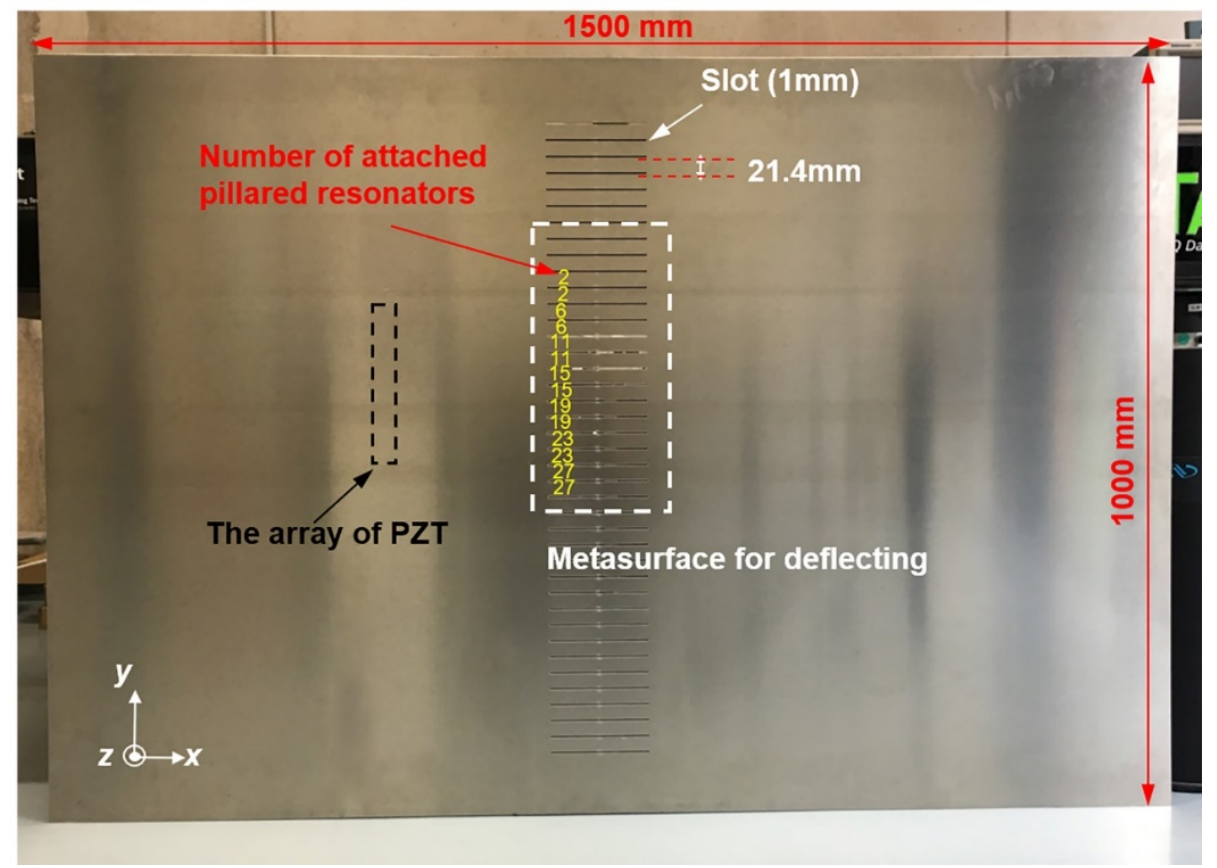

FIG. 6. Model diagram of the fabricated DEM with phase shift gradient is $d \phi / d y=0.5 k$.

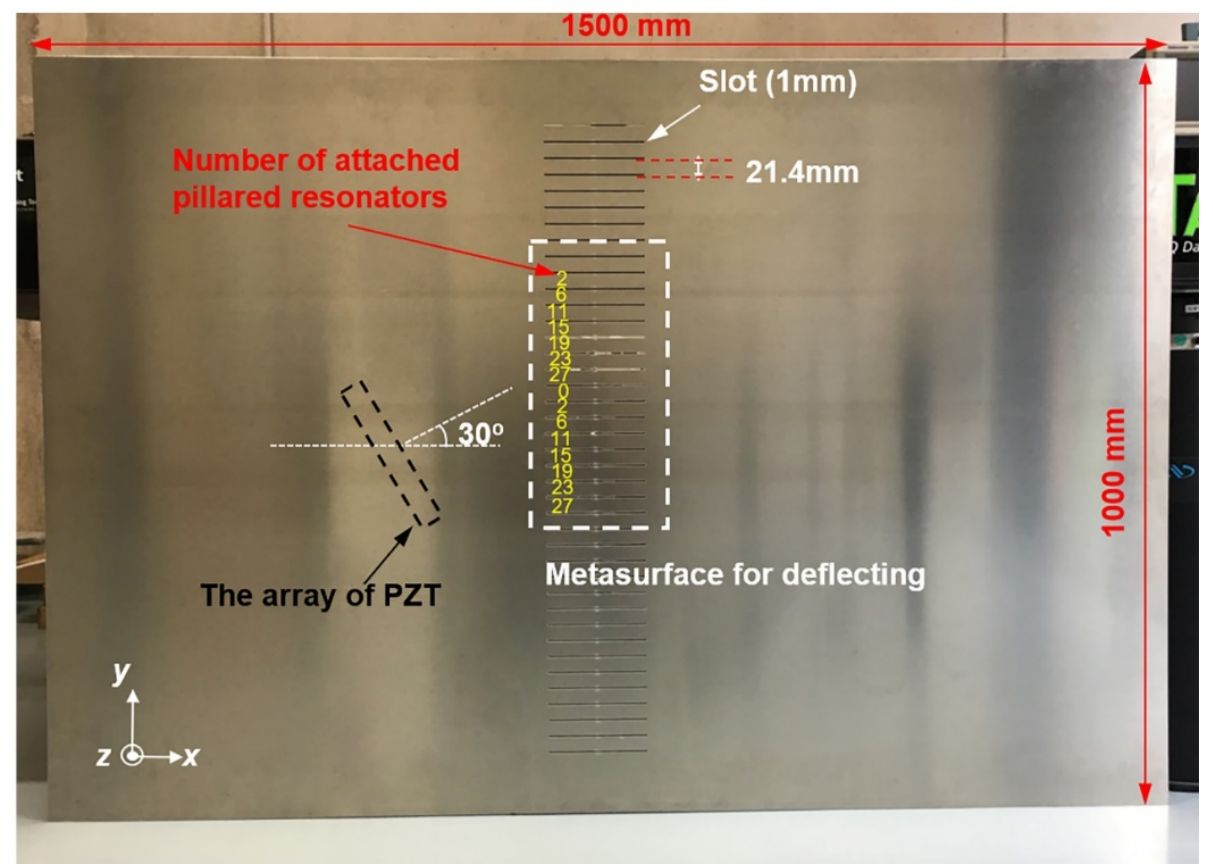

FIG. 7. Model diagram of the fabricated DEM with phase shift gradient is $d \phi / d y=k$. 


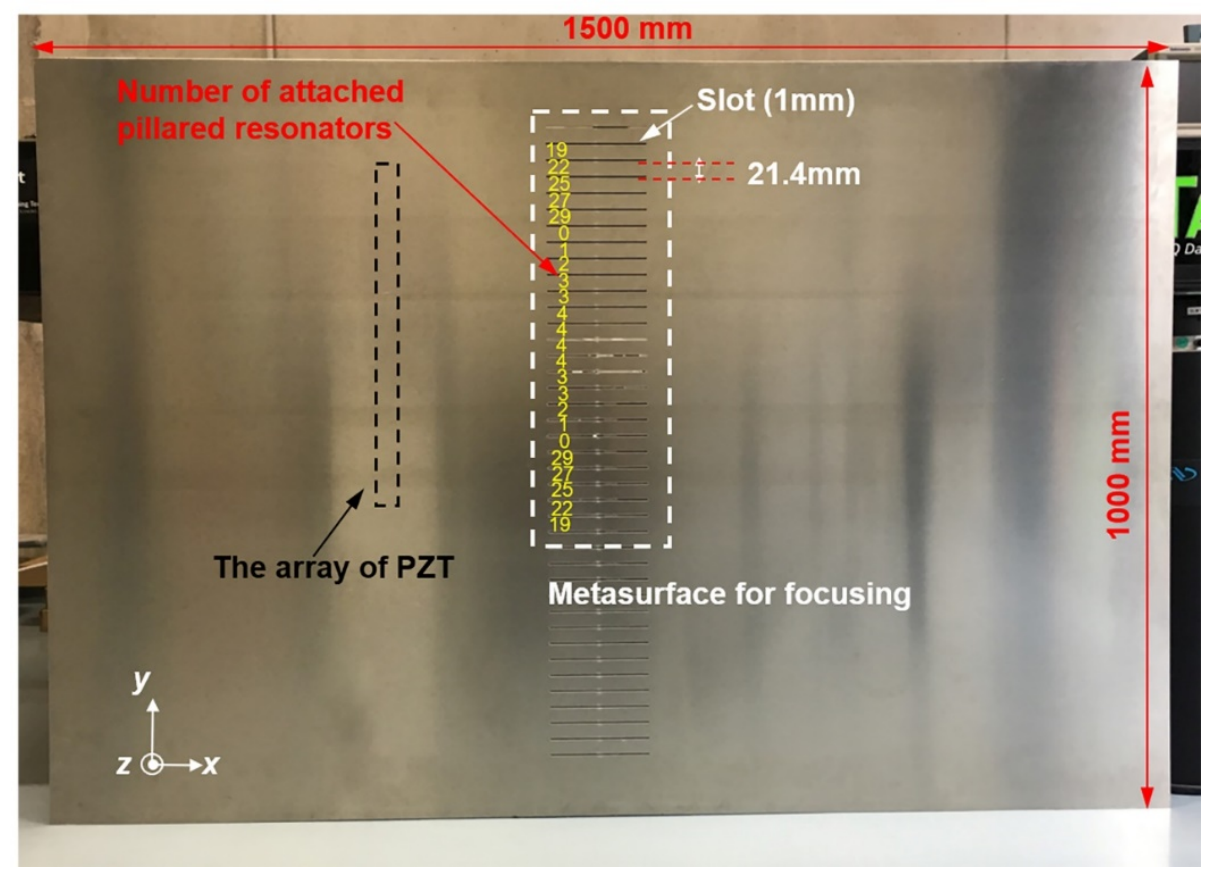

FIG. 8. Model diagram of the DEM with the desired focal distance $F=1.5 \lambda$.

An array of PZT-5A patches $(20 \mathrm{~mm} \times 20 \mathrm{~mm} \times 0.3 \mathrm{~mm})$ were bonded on the surface of the plate as actuators. The spacing of adjacent piezoelectric patches is the same as the piezoelectric patch width according to the phased array theory [12]. The widths of the DEMs only need to be larger than the width of the excited flexural beams. For simplicity, in the deflection experiments shown Fig. 6 and Fig. 7, we adopted 6 piezoelectric patches (the total width is $220 \mathrm{~mm}$ ) to excite flexural waves and 16 subunits to build the DEMs (the total width is $342.4 \mathrm{~m}$ ). In the focusing experiments shown Fig. 8, we adopted 12 piezoelectric patches (the total width is $460 \mathrm{~mm}$ ) to excite flexural waves and 24 subunits to build the DEM (the total width is $513.6 \mathrm{~mm}$ ). With the controlling of the waveform editor (based on Labview Software), a 5-cycle tone burst was generated by the signal generator (Agilent 33220A) and then amplified by a power amplifier (HVPA05). The wave fields in the incident and transmitted areas were measured by the measurement mode "time" of the PSV-400 scanning laser Doppler 
vibrometer [26].

\section{APPENDIX D: THE DESIGN OF THE RECONFIGURABLE SUBUNIT}

The schematic diagram of the reconfigurable subunit is shown in Fig. 9. It can be seen from Fig. 9 that the reconfigurable subunit has some improvements compared with the disordered subunit in Fig. 1(b). A thin layer of iron (about $0.1 \mathrm{~mm}$ ) is plated on the upper surface of the pillar by using a PVD system and magnetized as the N pole by annealing at $300^{\circ} \mathrm{C}$ for $2.0 \mathrm{~h}$ in a magnetic field of $1 \mathrm{~T}$ [28]. Three electromagnets are placed above the pillars. The electromagnets have magnetic poles that can convert $\mathrm{N}$ and $\mathrm{S}$ to each other on the upper and lower surfaces. A plastic guide rod is attached to the upper surface of the pillar, which can flexibly move the position of the pillar.

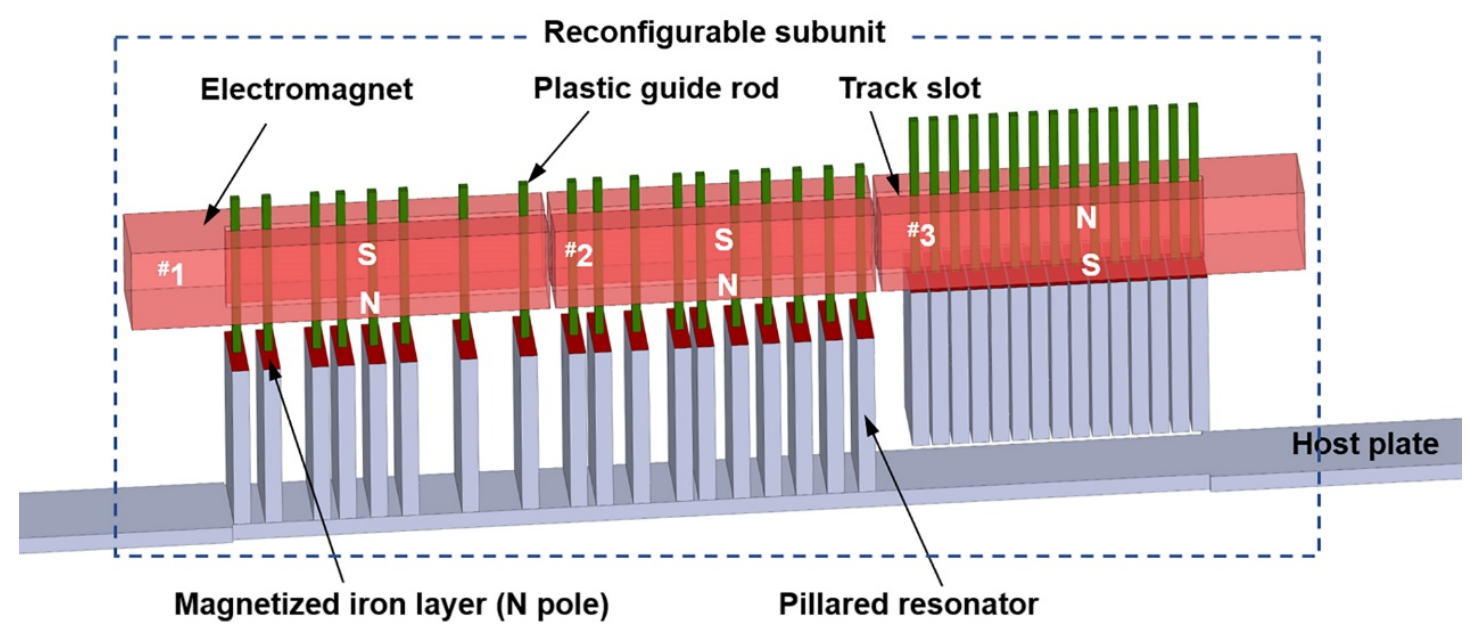

FIG. 9. Schematic diagram of the reconfigurable subunit

The working principle is very simple. Magnetic fields in different directions are formed under the three energized electromagnets. For example, magnetic fields are N, $\mathrm{N}$ and $\mathrm{S}$ poles for lower surfaces of ${ }^{\#} 1,{ }^{\#} 2$ and ${ }^{\#} 3$ electromagnets, respectively. We can move the pillar through the plastic guide rod to select the repulsion or attraction applied by the magnetic field. Under the ${ }^{\#} 1$ and ${ }^{\#} 2$ electromagnet, the pillar is repulsed and 
attached to the host plate. Under the ${ }^{\#} 3$ electromagnet, the pillar is separated from the host plate due to the magnetic attraction. Therefore, the number of pillars attached on the host plate can be reconstructed by moving the pillars to different magnetic fields. In this way, the disorder has great advantages over the order. The reason is the disorder releases the degree of freedom of pillar position, which greatly enhance reconfigurability.

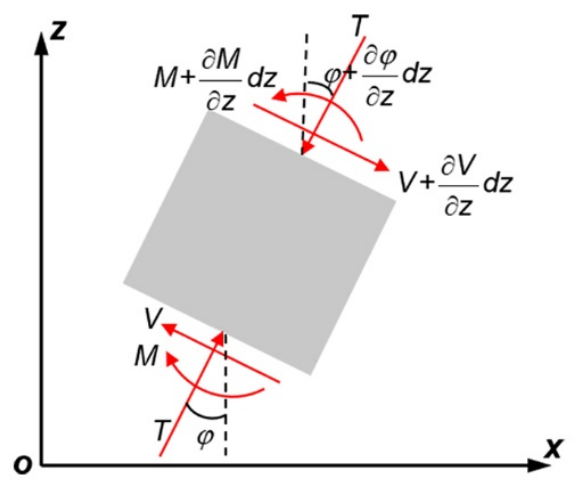

FIG. 10. The differential element of the pillar resonator.

We also have conducted the feasibility analysis for the designed structure. First, in the experiment, we can apply a small amount of oil on the lower surface of the pillar to fill the gap between the pillar and the host plate. This super subwavelength oil layer not only ensures a good connection between the pillar and the host plate, but also ensures that it does not affect the waves propagation at the joint interface. Then, the impedance of plastic and the thin iron layer is greatly mismatched, which will ensure that the lightweight plastic guide rod will not affect the wave propagation in the pillar. Finally, applying a repulsive force to the pillar is equivalent to giving the pillar an axial prestress $T$. The differential element of the pillar is taken for analysis, as shown in Fig. 10. The equation of motion in the $x$ direction, following Newton's second law ( $\Sigma F=m a$ ), 
is as follows:

$$
d_{2} \rho d z \frac{\partial^{2} w}{\partial t^{2}}=V+\frac{\partial V}{\partial z} d z-V+T \varphi-T\left(\varphi+\frac{\partial \varphi}{\partial z} d z\right)
$$

where $w$ is the displacement of the particle of the pillar in the $x$ direction. Substituting the relationships $\varphi=\partial w / \partial z$ and $V=E I \frac{\partial^{3} w}{\partial z^{3}}$ into Eq. (D1), we obtain

$$
E I \frac{\partial^{4} w}{\partial z^{4}}-T \frac{\partial^{2} w}{\partial z^{2}}-\omega^{2} \rho d_{2}=0
$$

From Eq. (D2), the flexural wavenumber can be obtained

$$
k_{b}^{*}= \pm \sqrt{\frac{T}{2 E I} \pm \sqrt{\left(\frac{T}{2 E I}\right)^{2}+\frac{\omega^{2} \rho d_{2}}{E I}}}
$$

where, $2 E I=353.5$. When $T /(2 E I)<0.06<<1$, we can get $k_{b}^{*} \approx k_{b 2}$. It indicates that the pre-pressure $[T /(2 E I)<0.06]$ does not affect the propagation of flexural waves in the pillar. Through the same analysis, we find the pre-stress $[T /(2 E I)<0.06]$ hardly affect the propagation of the longitudinal wave in the pillar and the propagations of the flexural wave and the longitudinal wave in the plate. Therefore, the pre-stress $[T /(2 E I)$ $<0.06$ ] does not affect the propagations of flexural waves and longitudinal wave, at the same time, it can make the pillar stably attached to the host plate.

It should be point that for the $x$-direction boundary condition at the junction of the pillar and the host plate, the non-glued pillar in the above reconfigurable subunit may weaken it compared with the glued pillar. We can reduce the height of the pillared resonator or decrease the rigidity of the pillared resonator, so that the vibration of the pillared resonator can couple into the host plate with the dominant effect coming from the compressional modes of the resonator [10,29], in other word, the $y$-direction 
boundary condition will be dominant at the junction of the resonator and the host plate. In this way, it can eliminate the impact of weakened $x$-direction boundary conditions.

\section{APPENDIX E: METHOD FOR NUMERICAL SIMULATIONS}

All the pillared resonators and plate are made of aluminum alloy, with the elastic

modulus $E_{\text {alu }}=70 \mathrm{GPa}$, Poisson's ratio $v_{\text {alu }}=0.33$, and the density $\rho_{\text {alu }}=2700 \mathrm{~kg} / \mathrm{m}^{3}$. The phase shift and transmission amplitude for the $2 \mathrm{D}$ subunits are obtained using COMSOL Multiphysics 5.4 software (Plane Strain Module). Perfect matched layers (PMLs) are used on both ends of every strip-like model to avoid any reflection from the boundaries. An exciting force is applied normally on the surface of the left part of the plate (at the point $\mathrm{P}$ in Fig. 1(c)). The phase shift and transmission amplitude of the excited flexural wave in the far field of the right part of the plate (at the point $\mathrm{Q}$ ) are examined by varying the number of resonators $j$ in the subunit. All the full-wave simulations for the metasurface are obtained using COMSOL Multiphysics 5.4 software (Solid Mechanics Module). For all simulations on the wave patterns of metasurfaces, PMLs are used on all outer boundaries.

\section{APPENDIX F: SIMULATIONS AND MEASUREMENTS FOR THE ORDERED METASURFACES}

The ordered elastic metasurface with the uniform pillars spacing of $5 \mathrm{~mm}$ is also constructed to achieve anomalous deflection and negative deflection. The full field experimental measurement is shown in Figs. 11(c) and 11(d). 
(a) Ordered pillars

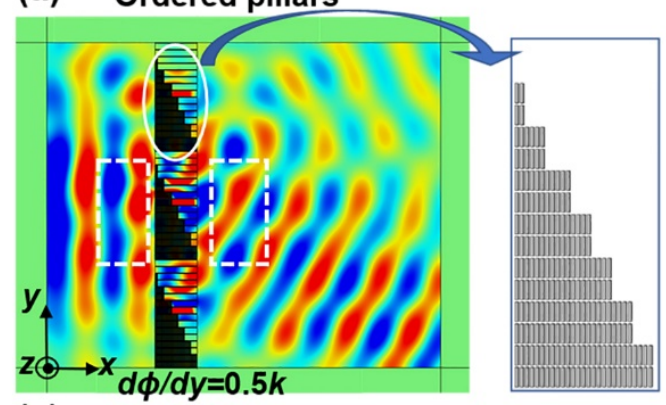

(c)

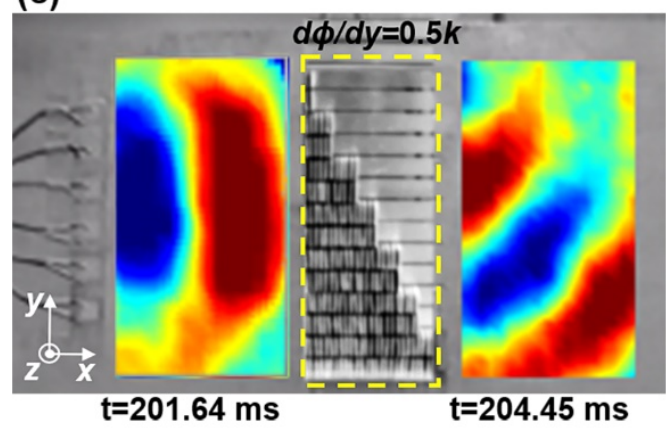

(b) Ordered pillars

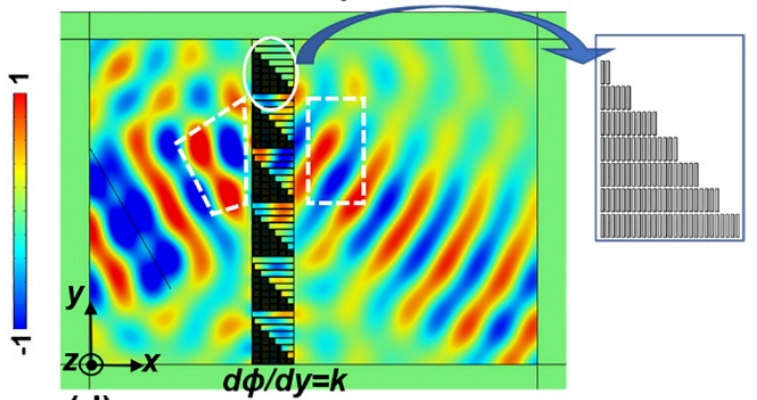

(d)

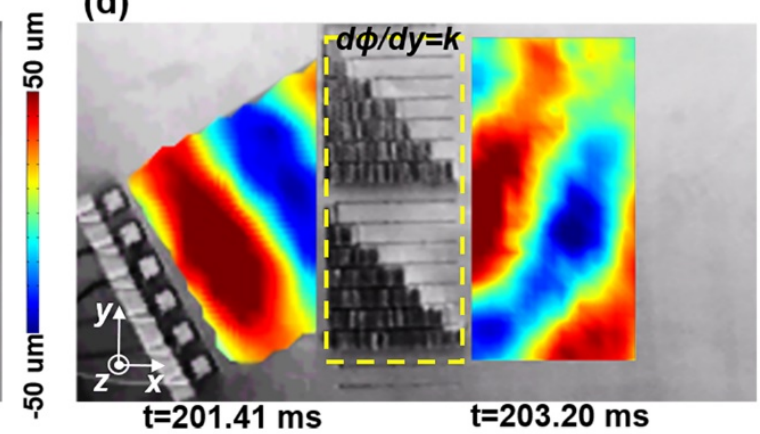

FIG. 11. (a) and (b) show wave fields for vertical and oblique incident flexural wave Gaussian beams pass through two ordered metasurfaces with phase shift gradients of $d \phi / d y=0.5 k$ and $d \phi / d y=k$, respectively. (c) and (d) show the full field experimental measurements.

The ordered elastic metasurface with the uniform pillars spacing of $5 \mathrm{~mm}$ is constructed to achieve flexural wave focusing. The full field experimental measurement is shown in Fig. 12(d). 
(a)

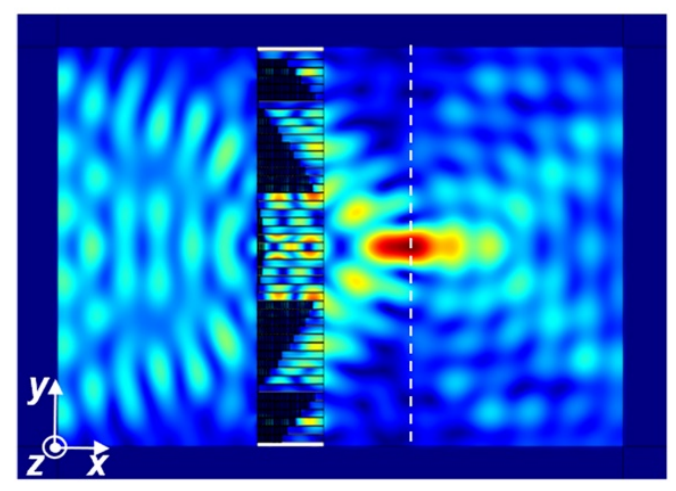

(c)

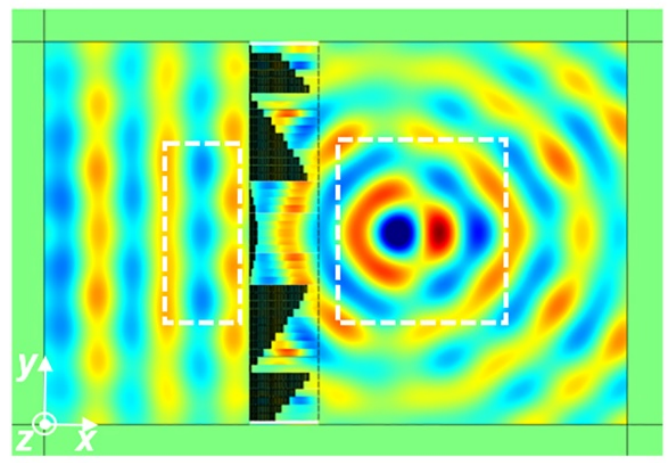

(b)

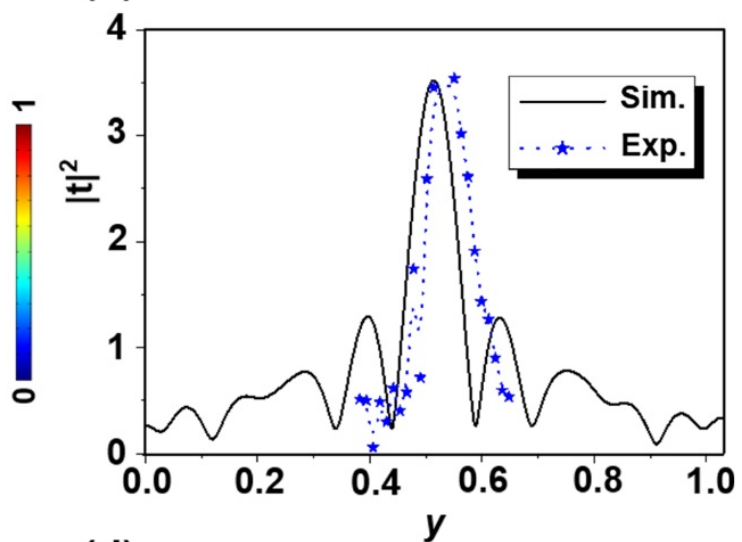

(d)

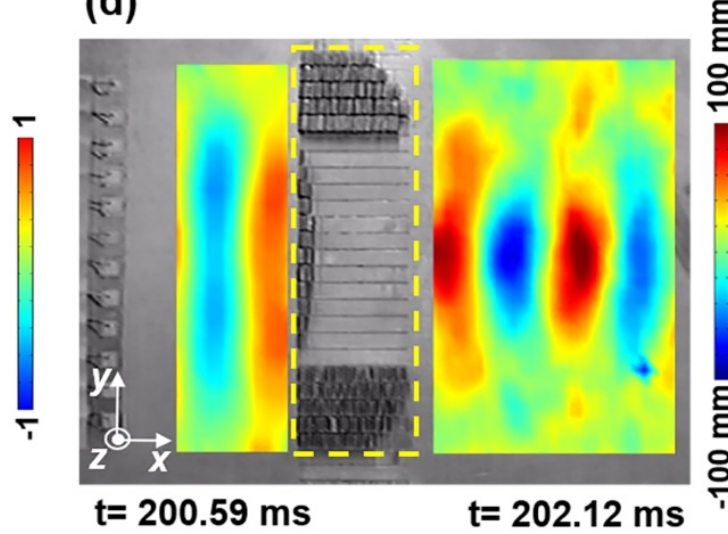

FIG. 12. (a) and (c) numerically show the transmitted intensity and displacement fields with focus at $1.5 \lambda$ for the ordered elastic metasurface, respectively. (b) provides the normalized simulated and experimental transmitted intensity at the focal spot along the $y$-direction $(x=1.5 \lambda)$. The experimental measurement is shown in (d). 


\section{REFERENCES}

[1] F. Baboux, L. Ge, T. Jacqmin, M. Biondi, E. Galopin, A. Lemaître, L. L. Gratiet, I. Sagnes, S. Schmidt, H. E. Türeci, A. Amo, J. Bloch, Bosonic Condensation and Disorder-Induced Localization in a Flat Band, Phys. Rev. Lett. 116, 066402 (2016).

[2] G. M. Conley, M. Burresi, F. Pratesi, K. Vynck, D. S. Wiersma, Light Transport and Localization in Two-Dimensional Correlated Disorder, Phys. Rev. Lett. 112, 143901 (2014).

[3] D. Nau, A. Schonhardt, C. Bauer, A. Christ, T. Zentgraf, J. Kuhl, M. W. Klein, H. Giessen, Correlation Effects in Disordered Metallic Photonic Crystal Slabs, Phys. Rev. Lett. 98, 133902 (2007).

[4] S. Hughes, L. Ramunno, J. F. Young, J. E. Sipe, Extrinsic Optical Scattering Loss in Photonic CrystalWaveguides: Role of Fabrication Disorder and Photon Group Velocity, Phys. Rev. Lett. 94, 033903 (2005)

[5] P. Celli, B. Yousefzadeh, C. Daraio, S. Gonella, Bandgap widening by disorder in rainbow metamaterials, Appl. Phys. Lett. 114, 091903 (2019).

[6] A. A. Asatryan, L. C. Botten, M. A. Byrne, V. D. Freilikher, S. A. Gredeskul, I. V. Shadrivov, R. C. McPhedran, Y.S.Kivchar, Suppression of Anderson Localization in Disordered Meta-materials, Phys. Rev. Lett. 99, 193902 (2007).

[7] V. Bellani, E. Diez, R. Hey, L. Toni, L. Tarricone, G. B. Parravicini, F. Domínguez-Adame, R. Gómez-Alcalá, Experimental Evidence of Delocalized States in Random Dimer Superlattices, Phys. Rev. Lett. 82, 2159 (1999).

[8] C. Liu, W. Gao, B. Yang, S. Zhang, Disorder-Induced Topological State Transition in Photonic Metamaterials, Phys. Rev. Lett. 119, 183901 (2017).

[9] A. Rahimzadegan, D. Arslan, R. N. S. Suryadharma, S. Fasold, M. Falkner, T. Pertsch, I. Staude, C. Rockstuhl, Disorder-Induced Phase Transitions in the Transmission of Dielectric Metasurfaces, Phys. Rev. Lett. 122, 015702 (2019).

[10] M. Rupin, F. Lemoult, G. Lerosey, P. Roux, Experimental Demonstration of Ordered and Disordered Multiresonant Metamaterials for Lamb Waves, Phys. Rev. Lett. 112, 234301 (2014).

[11] P. B. Catrysse, S. Fan, Routing of Deep-Subwavelength Optical Beams and Images without Reflection and Diffraction Using Infinitely Anisotropic Metamaterials, Adv. Mater. 25, 194 (2013). 
[12] R. Zhu, X. N. Liu, G. K. Hu, C. T. Sun, G. L. Huang, Negative refraction of elastic waves at the deep-subwavelength scale in a single-phase metamaterial, Nat. Commun. 5, 5510 (2014).

[13] J. M. Kweun, H. J. Lee, J. H. Oh, H. M. Seung, Y. Y. Kim, Transmodal Fabry-Perot Resonance: Theory and Realization with Elastic Metamaterials, Phys. Rev. Lett. 118, 205901 (2017).

[14] N. F. Yu, P. Genevet, M. A. Kats, F. Aieta, J. P. Tetienne, F. Capasso, Z. Gaburro, Light Propagation with Phase Discontinuities: Generalized Laws of Reflection and Refraction, Science 334, 333 (2011).

[15] E. Bok, J. J. Park, H. Choi, C. K. Han, O. B. Wright, S. H. Lee, Metasurface for Water-to-Air Sound Transmission, Phys. Rev. Lett. 120, 044302 (2018).

[16] B. Assouar, B. Liang, Y. Wu, Y. Li, J. Cheng, Y. Jing, Acoustic metasurfaces, Nat. Rev. Mater. 3, 460 (2018).

[17] Y. Li, C. Shen, Y. Xie, J. Li, W. Wang, S. A. Cummer, Y. Jing, Tunable asymmetric transmission via Lossy acoustic metasurfaces, Phys. Rev. Lett. 119, 035501 (2017).

[18] Y. Zhu, J. Hu, X. Fan, J. Yang, B. Liang, X. Zhu, J. Cheng, Fine manipulation of sound via lossy metamaterials with independent and arbitrary reflection amplitude and phase, Nat. Commun. 9, 1632 (2018).

[19] S. W. Fan, S. D. Zhao, A. L. Chen, Y. F. Wang, B. Assouar, Y. S. Wang, Tunable Broadband Reflective Acoustic Metasurface, Phys. Rev. Applied 11, 044038 (2019).

[20] H. Zhu, F. Semperlotti, Anomalous Refraction of Acoustic Guided Waves in Solids with Geometrically Tapered Metasurfaces, Phys. Rev. Lett. 117, 034302 (2016).

[21] Y. Q. Liu, Z. X. Liang, F. Liu, O. Diba, A. Lamb, J. S. Li, Source Illusion Devices for Flexural Lamb Waves Using Elastic Metasurfaces, Phys. Rev. Lett. 119, 034301 (2017).

[22] M. Jang, Y. Horie, S. M. Kamali, A. Shibukawa, A. Arbabi, J. Brake, H. Ruan, Y. Liu, A. Faraon, C. Yang, Wavefront shaping with disorder-engineered metasurfaces, Nat. Photonics 12, 84 (2018).

[23] Y. L. Xu, L. Y. Cao, Z. C. Yang, Deflecting incident flexural waves by nonresonant single-phase meta-slab with subunits of graded thicknesses, J. Sound. Vib. 454, 51 (2019).

[24] J. Zhang, X. Su, Y. L. Liu, Y. X. Zhao, X. Jing, N. Hu, Metasurface constituted by thin composite beams to steer flexural waves in thin plates, Int. J. Solids Struct. 162, 14 (2018).

[25] See Supplemental Material at [ ] for the detailed propagation phenomena for the anomalous deflections and focusing. 
[26] Y. B. Jin, E. El Boudouti, Y. Pennec, B. Djafari-Rouhani, Tunable Fano resonances of Lamb modes in a pillared metasurface, J. Phys. D. Appl. Phys. 50, 425304 (2017).

[27] L. Y. Cao, Z. C. Yang, Y. L. Xu, B. M. Assouar, Deflecting flexural wave with high transmission by using pillared elastic metasurface, Smart Mater. Struct. 27, 075051 (2018).

[28] Z. M. Zeng, P. Upadhyaya, P. K. Amiri, K. H. Cheung, J. A. Katine, J. Langer, K. L. Wang, H. W. Jiang, Enhancement of microwave emission in magnetic tunnel junction oscillators through in-plane field orientation, Appl. Phys. Lett. 99, 032503 (2011).

[29] D. J. Colquitt, A. Colombi, R. V. Craster, P. Roux, S. R. L. Guenneau, Seismic metasurfaces: Subwavelength resonators and Rayleigh wave interaction, J. Mech. Phys. Solids. 99(2017). 